\title{
Effects of desired speeds for queuing and delay on single-lane road segments
}

\author{
Mats Wiklund, Arne Carlsson, Olle Eriksson, Johan Olstam and Andreas Tapani
}

\author{
Linköping University Post Print
}

Tweet

N.B.: When citing this work, cite the original article.

This is an electronic version of an article published in:

Mats Wiklund, Arne Carlsson, Olle Eriksson, Johan Olstam and Andreas Tapani, Effects of desired speeds for queuing and delay on single-lane road segments, 2015, Transportmetrica A: Transport Science, (11), 8, 716-728.

Transportmetrica A: Transport Science is available online at informaworldTM:

http://dx.doi.org/10.1080/23249935.2015.1066462

Copyright: Taylor \& Francis: STM, Behavioural Science and Public Health Titles

http://www.tandf.co.uk/journals/default.asp

Postprint available at: Linköping University Electronic Press

http://urn.kb.se/resolve?urn=urn:nbn:se:liu:diva-120782 
To appear in Transportmetrica A: Transport Science

Vol. 00, No. 00, Month 20XX, 1-20

\title{
Effects of desired speeds for queuing and delay on single lane road segments
}

\author{
Mats Wiklund ${ }^{\mathrm{a}}$, Arne Carlsson ${ }^{\mathrm{b}}$, Olle Eriksson ${ }^{\mathrm{b}}$, Johan Olstam bc*, Andreas Tapani bc \\ ${ }^{a}$ Transport Analysis, Torsgatan 30, SE-113 21 Stockholm, Sweden; ${ }^{b}$ Swedish National Road and \\ Transport Research Institute (VTI), SE-581 95 Linköping, Sweden; ${ }^{c}$ Linköping University, \\ Department of Science and Technology (ITN), SE-601 74 Norrköping, Sweden
}

(2014-10-30, revised 2015-03-06)

To improve road safety on parts of the road network carrying low traffic volumes, road designs are proposed including single lane road segments and periodic overtaking lanes. These roads have been proven to contribute to substantial benefits in terms of road safety. However, overtaking of slower vehicles is only possible on segments including an overtaking lane and not on the single lane road segments. Driver and vehicle heterogeneity resulting in differences in desired speeds are consequently decisive for the traffic performance. Sufficient quality of service is relying on an appropriate design and distribution of single lane segments and overtaking lanes.

In this paper, we study the effect of the desired speed distribution on traffic performance on single lane road segments. Expressions are derived for the travel time, delay and percent time spent following. The derived expressions link the desired speed distribution, the single lane segment length and the traffic flow to the resulting traffic performance. The results are verified through comparison with measures based on microscopic traffic simulation. The conclusion is that there is a good agreement between derived measures and simulation results. The derived measures should therefore not only be of theoretical interest, but also of practical use to estimate traffic performance on single lane road segments.

Keywords: single lane road; overtaking restriction; percent time spent following; desired speed distribution

\footnotetext{
*Corresponding author. Email: johan.olstam@vti.se
} 


\section{Introduction}

In many places, extensive parts of the road network are carrying low traffic volumes. To improve road safety and traffic flow conditions on these links, road designs are proposed including single lane road segments and periodic overtaking lanes. Examples of such road designs include, 2+1-roads (Bergh, Carlsson, and Larsson 2003), two-lane expressways (Catbagan and Nakamura 2006) and super 2 highways (Brewer, Venglar, and Ding 2012). These road designs have been shown to result in superior road safety compared to single carriageway two lane roads (Bergh, Carlsson, and Larsson 2003; Park, Fitzpatrick, and Brewer 2012). A drawback is that overtaking of slower vehicles is only possible on segments including an overtaking lane and not on single lane road segments. Driver and vehicle heterogeneity resulting in differences in desired speeds are therefore decisive for the traffic performance on single lane road segments (as well as on other types of roads, see e.g. Treiber and Kesting (2013) for discussion and examples). Care should be taken to ensure sufficient quality of service when deciding on the distribution and lengths of single lane and overtaking lane road segments.

The design of roads including single lane road segments and periodic overtaking lanes are today mainly supported by traffic microsimulation studies (see e.g. Munehiro et al. 2012; Brewer, Venglar, and Ding 2012; Carlsson and Tapani 2005). As all microsimulation based analyses, these studies require substantial resources in terms of input data and time spent by the analysts. Few efforts to derive performance measures for these types of roads based on mathematical modelling have been found in the literature. Laval (2006) presented performance measures for two lane roads based on kinematic wave theory (Lighthill and Whitham 1955; Richards 1956) and moving bottlenecks (Newell 1998) and indicated how these measures could be applied to single lane road segments. However, traffic performance on single lane road segments will depend largely on the heterogeneity of the vehicles that make up the flow. Cowan $(1971,1980)$ recognized this and studied traffic properties on single lane roads based on a preferred travel time distribution. More recently, Shiomi, Yoshii, and Kitamura (2011) took a desired speed distribution as the starting point to study platoon formation and traffic breakdown at bottlenecks on single lane road segments.

In this paper, similar to Shiomi, Yoshii, and Kitamura (2011), we assume a distribution of desired speeds and study the effect of this distribution on traffic performance on single lane road segments. Main performance measures derived are travel time, delay and percent time spent following. Expressions relating the travel time and delay to a preferred travel time distribution have been presented by Cowan $(1971,1980)$. We derive results for single lane road segments that directly relate the desired speed distribution to the travel time, delay, time spent travelling at the desired speed and percent time spent following. To verify the results, we conduct a set of traffic simulation experiments and compare the derived measures to measures based on the output of the simulations. The derived expressions illustrate the effect of the assumptions made for the desired speed distribution, the single lane segment length and the traffic flow on the resulting traffic performance.

The paper is organised as follows. Section 2 provides a background to traffic performance estimation for roads including single lane road segments and periodic overtaking lanes. Section 3, is dedicated to the derivation of performance measures based on the desired speed distribution. Numerical experiments are presented in Section 4, comparing the derived measures to measures based on traffic simulation. Section 5 concludes the paper with a discussion on the results. 


\section{Background}

Measures commonly used to describe performance of road traffic facilities in terms of efficiency include speed, travel time and delay (Transportation Research Board 2010). Delay measures involve both the delay caused by the design of the infrastructure and the delay inflicted by the traffic. For roads with limited possibilities for overtaking, e.g. two lane highways, the perceived quality of service can be assumed to depend on the time spent following slower vehicles. Percent time spent following is therefore suggested as an additional performance measure for such roads.

To assess the performance of already existing infrastructure under the prevailing traffic conditions, the current practice relies on point based observations, i.e. speed and flow can be observed directly. Travel time is often derived by comparing the times that unique vehicles are observed at different positions. Due to the inherent difficulties associated with observing measures that require continuous collection of data over a distance or in time, the percentage of headways less than $3 \mathrm{~s}$ is suggested as a surrogate measure of percent time spent following (Luttinen 2001; Transportation Research Board 2010).

For infrastructure and traffic planning purposes, the current state of practice for traffic performance estimation is traffic simulation modelling (Barceló 2010). The strength of the simulation based approach to traffic performance estimation lies largely in its flexibility, allowing inclusion of different aspects of traffic heterogeneity, e.g. the distribution of desired speeds, and in simulation being a natural framework for modelling traffic dynamics. However, applications of traffic simulation are often resource demanding and the model complexity also makes it difficult to determine causal relationships between variables in the simulation. Such causal relationships can be derived based on mathematical modelling for simple traffic situations.

Early attempts at mathematical modelling of traffic on roads with limited possibilities for overtaking include steady state queuing models for traffic on two lane roads (see e.g. Galin (Goldfarb) and Epstein 1974; Daganzo 1975; Brilon 1977). Assumptions made in these early models were often that overtaking is allowed at all points along the road or that the traffic consists of a limited number of vehicle types traveling at vehicle type specific fixed speeds. Exceptions include the works of Cowan $(1971,1980)$ in which properties of traffic on single lane roads are studied based on a preferred travel time distribution. To predict traffic dynamics, models based on the kinematic wave theory proposed by Lighthill and Whitham (1955); Richards (1956) have proven successful. For traffic on roads with limited possibilities for overtaking Newell (1998) proposed a framework with slow moving vehicles as moving bottlenecks in a kinematic wave model and Laval (2006) derived performance measures for two lane roads based on Newell's theory.

Kinematic wave based traffic models describe the dynamics of the average traffic state based on specified fundamental diagrams. Besides the works of Cowan $(1971,1980)$ there are few not simulation based models in the literature that link traffic heterogeneity, due to e.g. differences between types of vehicles and in driver preferences, to the resulting traffic performance on roads with limited opportunities for overtaking. Cowan (1971, 1980) study the effect of preferred travel times and arrival headways on actual travel times, delays and the platoon formation process on single lane roads. The need to take into account traffic heterogeneity was also recognised by Shiomi, Yoshii, and Kitamura (2011) to motivate the derivation of a platoon formation model based on a desired speed distribution. Shiomi, Yoshii, and Kitamura (2011) assume platoon formation on single lane road segments due to the desired speed distribution and the vehicle interarrival times and derive a model for the formation probability of a platoon of specific size and specific speed of the leader vehicle. The platoon formation model derived is applied for 
subsequent modelling and analysis of breakdown probability at bottlenecks on single lane road segments.

The desired speed distribution can be described as a result of the preferences of the drivers in combination with the properties of the vehicles. Procedures for estimation of the desired speed distribution in real traffic have been studied by e.g. Branston (1979); Hoogendoorn (2005); Catbagan and Nakamura (2008). In the following section, we assume a given desired speed distribution and derive performance measures for single lane road segments that link this desired speed distribution, the length of the single lane segment and the traffic flow to the traffic performance. (20)

西

.




\section{Traffic performance on single lane road segments}

The performance measures derived in this paper are travel time, delay and percent time spent following. We start the presentation by deriving expressions for expected time spent travelling at the desired speed and expected delay. These expressions are both derived in three steps, namely to study a vehicle with a fixed speed when other vehicles also have a fixed speed, to generalise to a distribution of speeds for other vehicles and finally to generalise to a distribution of speeds for the studied vehicle. Total travelling time and percent time spent following are later on found as formulas including these derived measures.

Let a flow of vehicles arrive with rate (number per time unit) $q$ at a road segment of infinite length with unlimited possibilities for overtaking. Assume that the time between vehicle arrivals is exponentially distributed and that each vehicle travels at their desired speed. Consider a vehicle with given arrival time and speed $v$. Let $f$ be the probability density function for the speeds of vehicles arriving at the segment with density 0 for non-positive speeds. The concentration (number per distance unit) of vehicles with a specific speed $u$ along the segment depends on the arrival rate and the travel time per distance unit,

$$
\frac{q \cdot f(u)}{u} .
$$

The considered vehicle overtakes vehicles travelling at speed $u<v$ with rate $(v-u) \frac{q \cdot f(u)}{u}$ and concentration

$$
\frac{v-u}{v} \cdot \frac{q \cdot f(u)}{u}=q \cdot\left(\frac{1}{u}-\frac{1}{v}\right) f(u) .
$$

The distance to where this vehicle overtakes a first vehicle travelling at speed $u<v$ is exponentially distributed (see Appendix A for details). The distance to where this vehicle overtakes the first vehicle with any speed $u<v$ follows the distribution of the minimum of a set of independent exponential distributions. Such a minimum is also exponentially distributed (see Appendix A for details). The concentration of overtakings, $h(v, q)$, is given by the integral of the concentrations over all $u<v$,

$$
h(v, q)=q \int_{0}^{v}\left(\frac{1}{u}-\frac{1}{v}\right) f(u) d u,
$$

and the exponential probability density for the distance to the first overtaking, $l$, is

$$
h(v, q) e^{-l \cdot h(v, q)} .
$$

Now, consider the segment to have no possibilities for overtaking, which implies formation and concatenation of platoons. Then, if vehicles are assumed to have zero length, adapt their speed instantaneously when joining a platoon and travel with zero headway when in a platoon, $l$ becomes the distance travelled at the desired speed. Also, let the segment length be limited to $L$. This is equivalent to observing only a first part of length $L$ of a segment of infinite length. Consequently, Equation 1 applies but the vehicle travelling at speed $v$ is constrained by a slower vehicle only if $l<L$. The expected distance travelled at the desired speed therefore becomes the expectation of the minimum of $l$ 
and $L$,

$$
\begin{aligned}
\int_{0}^{\infty} \min (l, L) h(v, q) e^{-l \cdot h(v, q)} d l & =\int_{0}^{L} l \cdot h(v, q) e^{-l \cdot h(v, q)} d l+\int_{L}^{\infty} L \cdot h(v, q) e^{-l \cdot h(v, q)} d l \\
& =\frac{1-e^{-L \cdot h(v, q)}}{h(v, q)}
\end{aligned}
$$

The expected distance travelling at desired speed for a randomly chosen vehicle with any speed $v \sim f$ is

$$
\int_{0}^{\infty} \frac{1-e^{-L \cdot h(v, q)}}{h(v, q)} f(v) d v
$$

and the expected time spent travelling at the desired speed, $\phi$, becomes

$$
\phi=\int_{0}^{\infty} \frac{1-e^{-L \cdot h(v, q)}}{h(v, q)} \cdot \frac{f(v)}{v} d v
$$

To find expressions for travel time and the expected delay, consider a vehicle with given arrival time and speed $v$. Because every vehicle is assumed to travel at their desired speed before arriving at the segment, the time from the arrival of the last vehicle with speed $u$ until the arrival of the considered vehicle is exponentially distributed. Assume it arrived a time $a$ earlier. If all slower vehicles have the same desired speed $u$, the travel time for the considered vehicle becomes

$$
\max \left(\frac{L}{v}, \frac{L}{u}-a\right)
$$

where $a$ is exponentially distributed with rate $q_{u}$, the same as the arrival rate of slower vehicles. Let the random variable $R, \frac{1}{v} \leq R \leq \frac{1}{u}$ be the travel time for the vehicle with desired speed $v$. Also, let $G$ be the cumulative distribution function for $R$,

$$
G(r)=\left\{\begin{array}{cr}
0 & r<L / v \\
G^{*}(r) & L / v \leq r<L / u \\
1 & L / u \leq r
\end{array}\right.
$$

where $G^{*}(r)=P\left(\max \left(\frac{L}{v}, \frac{L}{u}-a\right) \leq r\right)$ which is $P\left(\frac{L}{u}-a \leq r\right)=P\left(a \geq \frac{L}{u}-r\right)$ for $L / v \leq r$. This probability is one minus the cumulative density at $(L / u-r)$ of an exponential with rate $q_{u}, \exp \left(-q_{u} \cdot\left(\frac{L}{u}-r\right)\right)$ for $r<\frac{L}{u}$.

Assume that all vehicles that have already entered the segment have a distribution of desired speeds $u$ and that this distribution is discrete with probability function $p(u)$. If the concidered vehicle can be constrained by any slower vehicle with a speed in the distribution $u$, then

$$
G(r)=\left\{\begin{array}{clrl}
0 & & r<L / v \\
G^{*}(r) & L / v & \leq r<L / \min (u) \\
1 & L / \min (u) \leq r
\end{array}\right.
$$


where $G^{*}(r)=\prod_{u<\frac{L}{r}} \exp \left(-q \cdot p(u)\left(\frac{L}{u}-r\right)\right)=\exp \left(-\sum_{u<\frac{L}{r}} q \cdot p(u)\left(\frac{L}{u}-r\right)\right)$. This means that for a continuous $u, G^{*}(r)=\exp \left(-\int_{0}^{\frac{L}{r}} q \cdot f(u)\left(\frac{L}{u}-r\right) d u\right)$ and that $G(r)$ is the probability that the travel time for a vehicle with desired speed $v$ becomes less than or equal to $r$ when taking into account all other vehicles that have entered the section with a continuous speed distribution.

The travel time has density $g(r)=\frac{d}{d r} G(r)=\lim _{\epsilon \rightarrow 0^{+}} \frac{G(r+\epsilon)-G(r)}{\epsilon}$ in $r>\frac{L}{v}$. Also, the delay is $r-\frac{L}{v}$ on that interval. The expected delay for the considered vehicle with speed $v$ is given by

$$
\int_{\frac{L}{v}}^{\infty}\left(r-\frac{L}{v}\right) g(r) d r=\lim _{\epsilon \rightarrow 0^{+}} \int_{\frac{L}{v}}^{\infty}\left(r-\frac{L}{v}\right) \frac{G(r+\epsilon)-G(r)}{\epsilon} d r .
$$

For a vehicle with any speed $v \sim f$, let $G_{v}$ be $G$ with a particular $v$. The expected delay, $\delta$, becomes

$$
\delta=\int_{0}^{\infty}\left(\lim _{\epsilon \rightarrow 0^{+}} \int_{\frac{L}{v}}^{\infty}\left(r-\frac{L}{v}\right) \frac{G_{v}(r+\epsilon)-G_{v}(r)}{\epsilon} d r\right) f(v) d v
$$

Given the expected delay, $\delta$, the expected travel time, $\tau$, can be obtained adding the expected travel time if all vehicles are assumed to travel at their desired speed throughout the road segment, i.e.

$$
\tau=\delta+L \int_{0}^{\infty} \frac{f(v)}{v} d v
$$

Finally, percent time spent following can be calculated as

$$
\frac{\tau-\phi}{\tau} \cdot 100
$$




\section{Numerical experiments}

The assumptions made in the derivation of the performance measures can be summarised as follows. Vehicles travelling at their desired speed enter a single lane segment with exponentially distributed inter-arrival times. We treat the vehicles as particles with no extension in space and the headway between vehicles travelling in a platoon is neglected. Also vehicles are assumed to maintain their desired speeds until they get constrained by a slower platoon and then instantaneously reduce their speed to the speed of the platoon.

To verify the performance measures derived and to analyse consequences of the assumptions made, numerical experiments using microscopic traffic simulation have been conducted. This approach allowed systematic analysis of the derived measures for different desired speed distributions, single lane segment lengths and traffic flows.

\section{1. $\quad$ Method}

The desired speed is in the experiments assumed to have normal distribution with expectation $\mu$ and standard deviation $\sigma$, i.e. the probability density for speed $u$ is

$$
f(u)=\frac{1}{\sigma \sqrt{2 \pi}} \exp \left(-\frac{1}{2}\left(\frac{u-\mu}{\sigma}\right)^{2}\right) \text {. }
$$

In the examples we truncate the distribution at 2.5 standard deviations, this apply both for the calculations using the derived measures and the simulations. It is the standard deviation before truncation, $\sigma$, that is discussed in the following text and figures. The standard deviation in the truncated distribution is about $0.95 \cdot \sigma$. The integrals in Equation 2 to 4 are approximated numerically using standard software's for numerical integration.

The traffic simulation experiments have been conducted using the Intelligent Driver Model (IDM; Treiber, Hennecke, and Helbing 2000). IDM is a widely used and referenced model that has been found to reproduce real traffic data with similar accuracy as other state of the art car-following models (Brockfeld, Kühne, and Wagner 2005; Punzo and Simonelli 2005). The IDM governs the longitudinal movements of a simulated vehicle through an acceleration rate specified with respect to the desired speed and the distance to the preceding vehicle.

In the applied traffic generation process vehicles are generated with exponentially distributed inter-arrival times to the simulated road. However, new vehicles may have to wait and adapt their speed until they do not need to decelerate more than $0.1 \mathrm{~m} / \mathrm{s}^{2}$ before they are allowed to enter the simulation. This is verified using the IDM. The simulations only included passenger cars, all with a length of $4.5 \mathrm{~m}$. All cars were controlled by an IDM with parameter values according to table 2 in Kesting et al. (2007), which e.g. imply a jam distance of $2 \mathrm{~m}$, a desired following time gap of $1.5 \mathrm{~s}$, a maximum acceleration of $1.4 \mathrm{~m} / \mathrm{s}^{2}$ and a desired deceleration of $2 \mathrm{~m} / \mathrm{s}^{2}$.

Percent time spent following have been calculated for the simulation results as the part of the travel time that the vehicles can be considered to follow the preceding vehicle. We have classified vehicles with time headway of less than $3 \mathrm{~s}$ as 'following', as suggested by Luttinen (2001) and in HCM 2010 (Transportation Research Board 2010).

\subsection{Comparison of derived measures and simulation results}

The effects of the standard deviation of the desired speed distribution, the single lane segment length and the traffic flow on the derived performance measures are studied in 
this section.

First, we focus on the effects of the standard deviation of the desired speed distribution. Consider a $2 \mathrm{~km}$ single lane road segment and let the arriving flow be 200 vehicles $/ \mathrm{h}$. Figure 1 to 3 display performance measures given by Equation 2 to 4 and the traffic simulation setup for different standard deviations of the desired speed distribution. The simulation results are based on ten simulation replications.

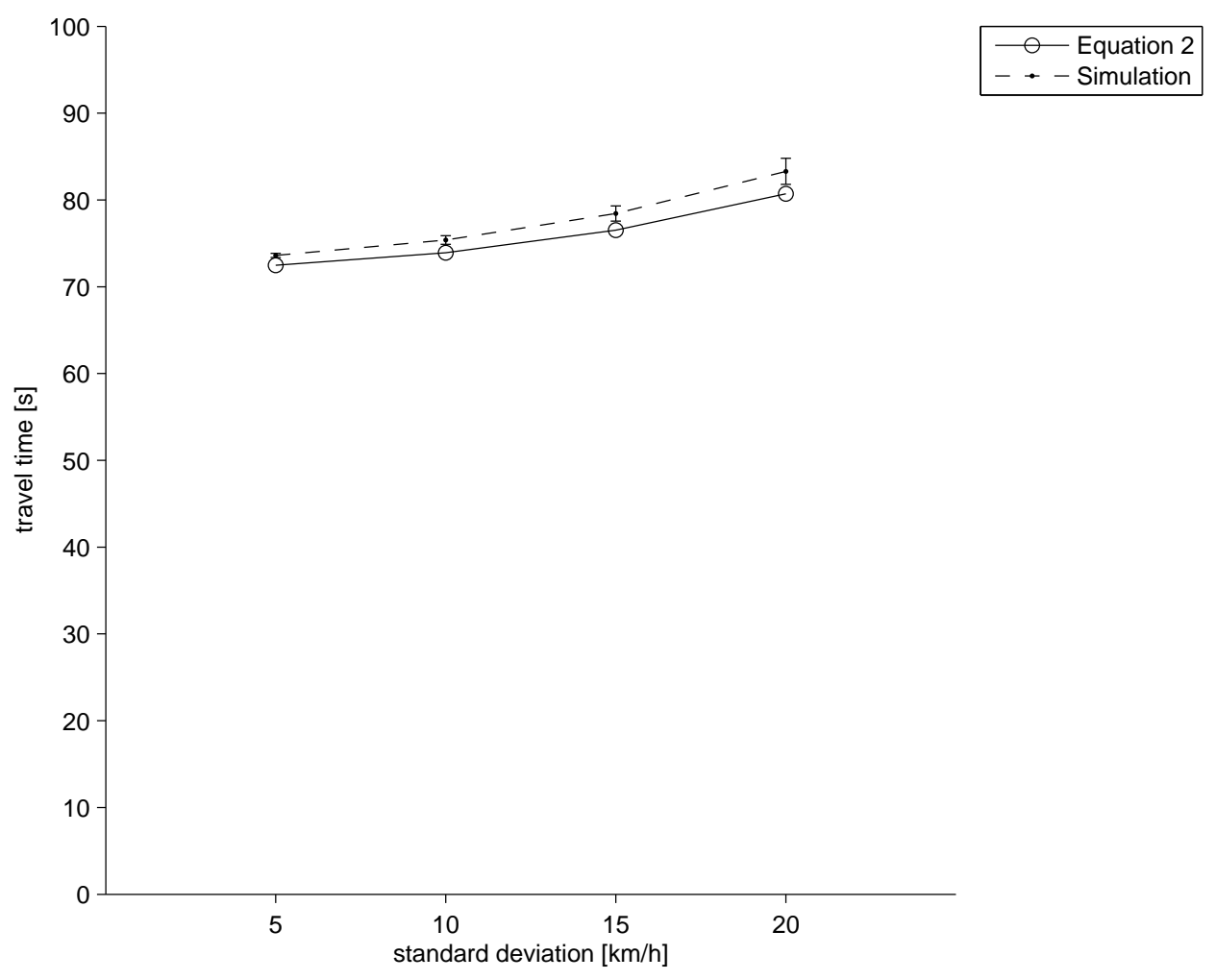

Figure 1. Travel time given by Equation 2 and the simulation results. Error bars for the simulation results indicate $95 \%$ confidence intervals (based on 10 simulation replications).

As can be seen in the figures there is a good agreement between the derived measures and the simulation results. It can be noted that the delay seems to be slightly underestimated (about $1 \mathrm{~s}$ ) by Equation 3. The assumptions of zero vehicle length and zero headway between vehicles in a platoon (see Section 3) contribute to this effect. Platoons will therefore have no extension in space, leading to a longer time until a vehicle catches up with a slower vehicle and therefore less delay.

To study the effect of the single lane segment length and the traffic flow on travel time, delay and percent time spent following, we compare the derived measures to the results of traffic simulations for the following combinations of single lane segment length and traffic flow:

- Single lane segment length, $L: 1,2,4,8 \mathrm{~km}$

- Flow, $q$ : 20, 100, 200, 400, 800 vehicles/h

The desired speed distribution is for all combinations above, a truncated normal distribution with mean and standard deviation $100 \mathrm{~km} / \mathrm{h}$ and $15 \mathrm{~km} / \mathrm{h}$, respectively. Figure 4 shows the average travel time for the 20 different combinations of single lane segment length and flow. As can be seen in the figure, there is, in general, a good agreement be- 


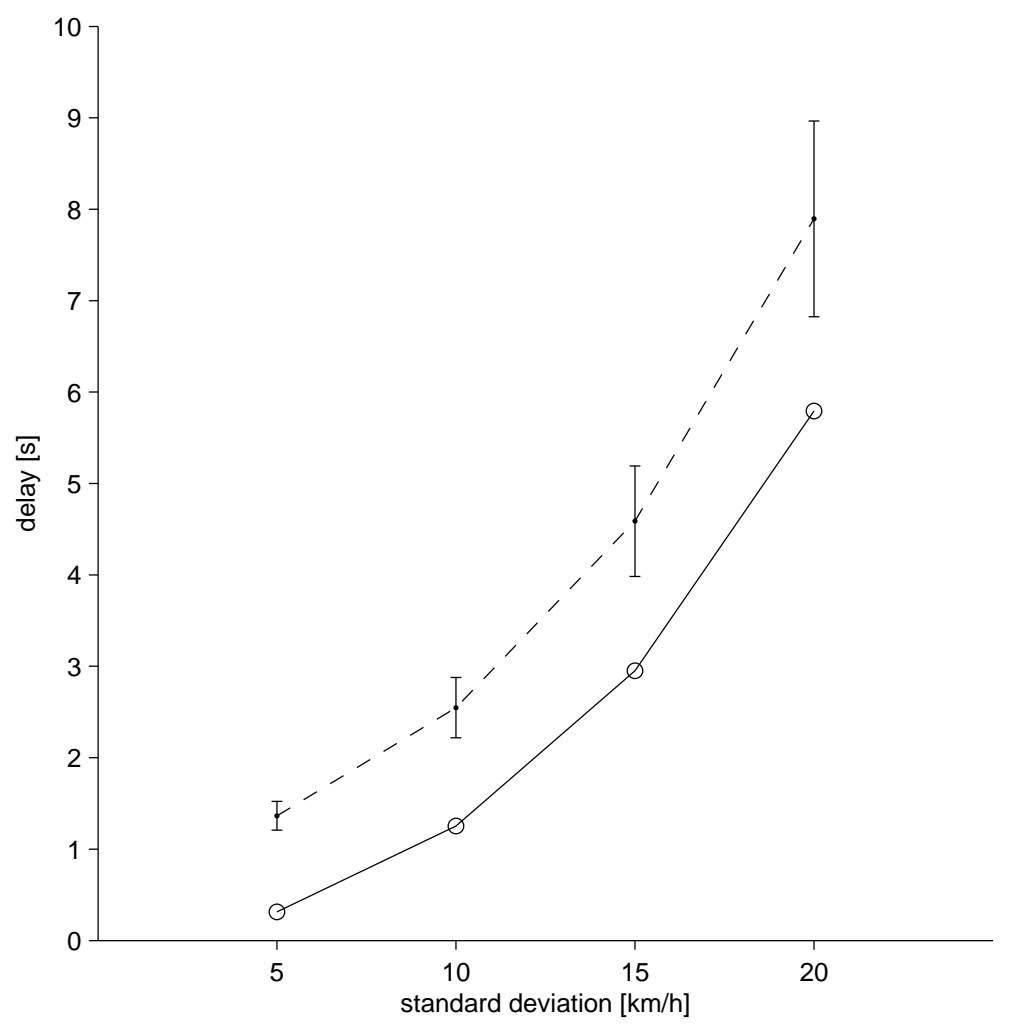

Figure 2. Delay given by Equation 3 and the simulation results. Error bars for the simulation results indicate $95 \%$ confidence intervals (based on 10 simulation replications).

tween the travel time given by Equation 2 and the simulation results. However, there is a tendency for larger differences between Equation 2 and the simulation results for the 400 and 800 vehicles/h flow levels compared to the lower flow levels. This difference is more pronounced in Figure 5 showing the delay given by Equation 3 and the simulation results. The differences observed for delay and travel time are partly due to the assumption of independent arrivals to the single lane road segment becoming questionable in higher flow conditions. The higher the flow the larger the proportion of vehicles already following a slower vehicle at the start of the segment. In addition, the effect of the assumptions of zero vehicle length and zero headway between vehicles in a platoon is naturally larger the shorter the considered segment. For example, a 3 second time headway with a speed of $27.8 \mathrm{~m} / \mathrm{s}$ gives a headway of $83.3 \mathrm{~m}$ which is $8.3 \%$ of $1 \mathrm{~km}, 4.2 \%$ of $2 \mathrm{~km}$ and so forth. This implies that a vehicle that caught up with another vehicle during the simulation did it in average $3 \mathrm{~s}$ or $83 \mathrm{~m}$ earlier than assumed in the derived measures. The conclusion is therefore better agreement between the derived measures and the simulation results the longer the segment length and the lower the traffic flow.

Figure 6 shows percent time spent following given by Equation 4 and the simulation results. There is an increasing difference between the derived percent time spent following measure and the simulation results for higher flow and shorter single lane segment lengths. Similarly as for travel time and delay, the assumptions made regarding independent arrivals to the start of the single lane segment, zero vehicle length and zero headway between vehicles in a platoon contribute to the differences observed. 


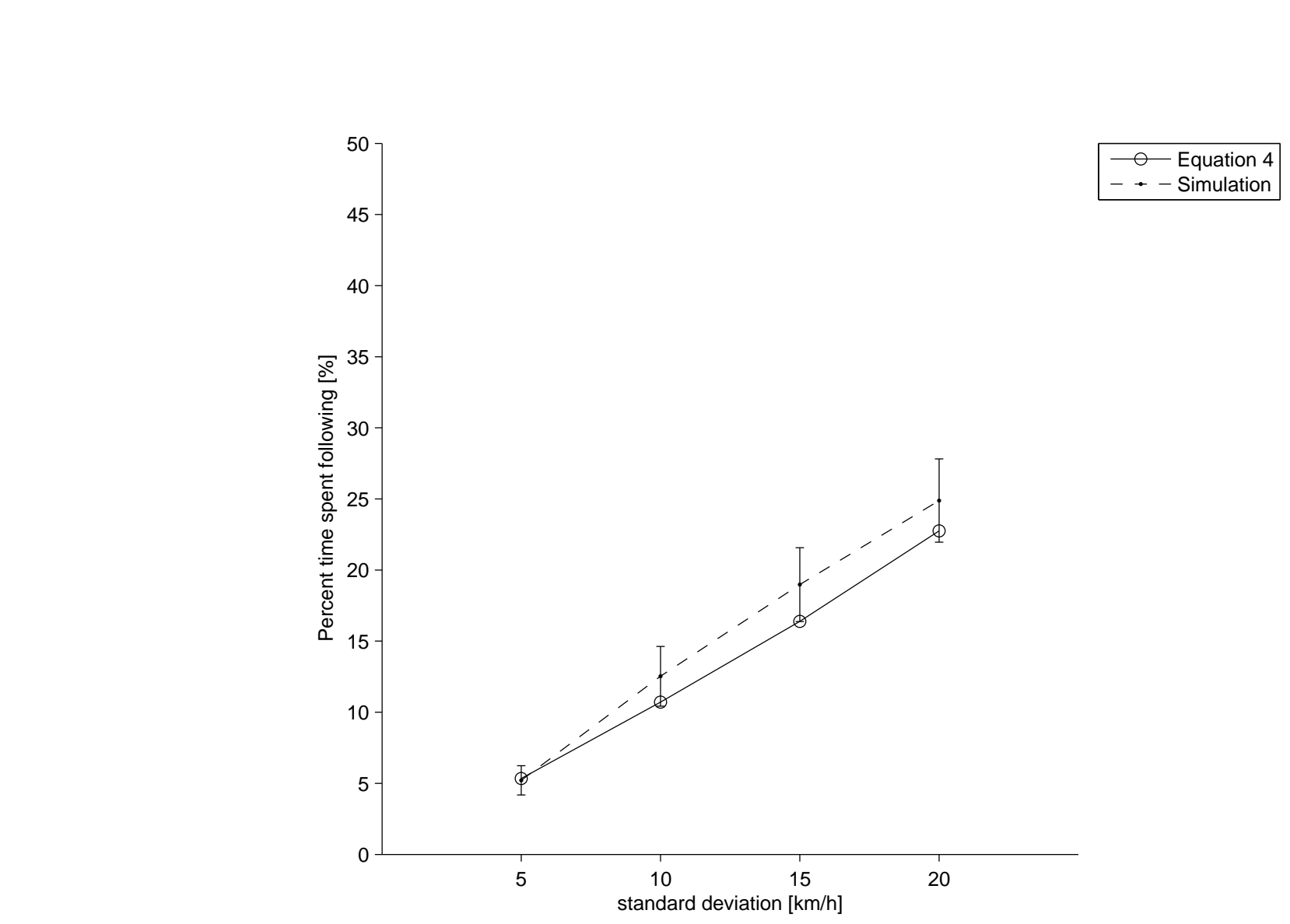

Figure 3. Percent time spent following (PTSF) given by Equation 4 and the simulation results. Error bars for the simulation results indicate $95 \%$ confidence intervals (based on 10 simulation replications). 

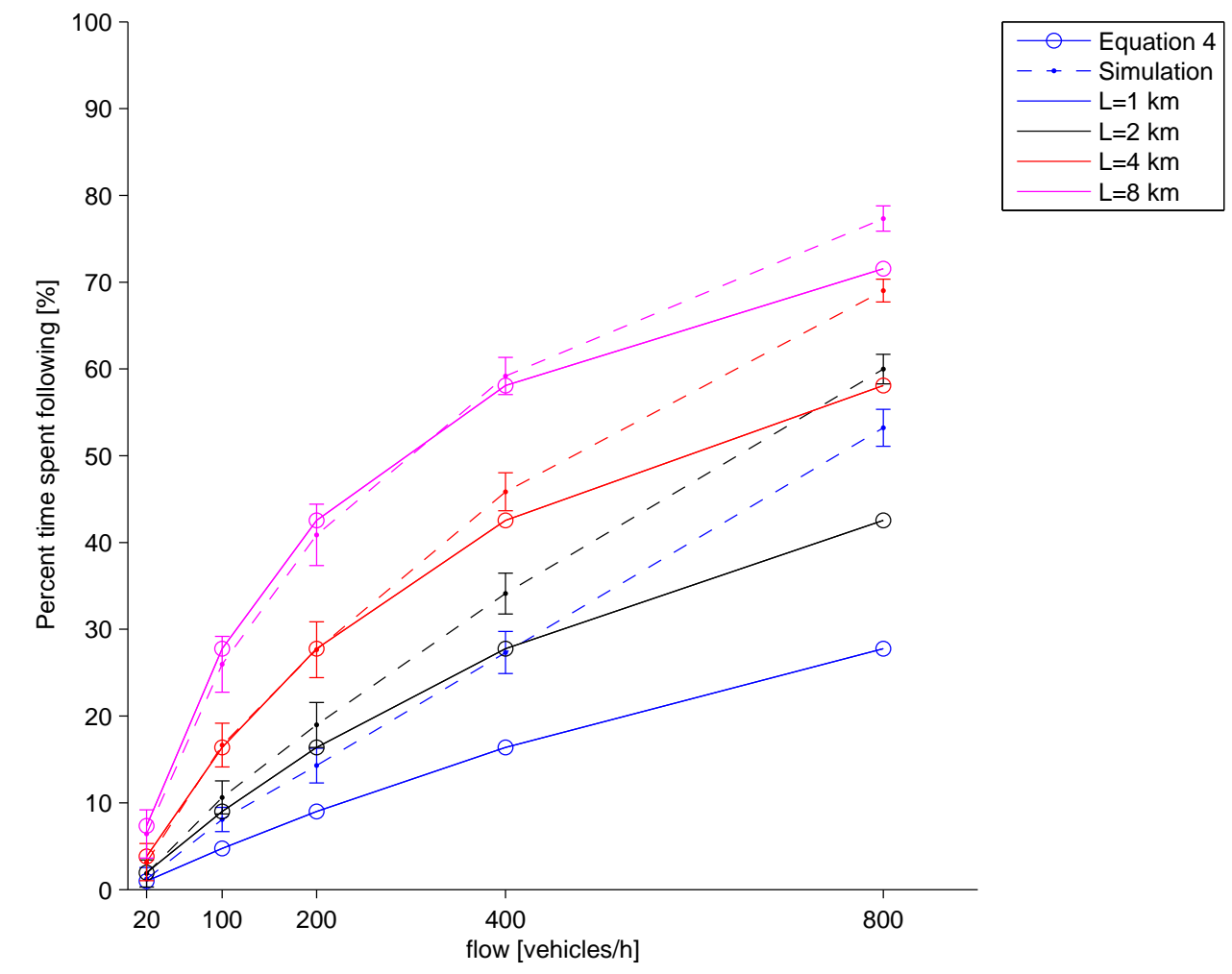

Figure 6. Percent time spent following (PTSF) given by Equation 4 and the simulation results. Error bars for the simulation results indicate $95 \%$ confidence intervals (based on 10 simulation replications).

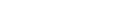

\begin{abstract}
the sinthtin rests indicate $95 \%$ confing
\end{abstract}

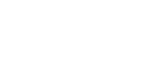




\section{Conclusions}

Performance measures have been derived that link the heterogeneity of the traffic flow, in the form of the desired speed distribution, to the resulting traffic condition on single lane road segments. Derived measures are travel time, delay and percent time spent following. The expressions obtained can be solved with numerical methods. The desired speed distribution was in the numerical experiments assumed to be a truncated normal density, but there are no restrictions imposed on the desired speed distribution, i.e. the density for any positive valued random variable can be used..

We verify the results through a set of experiments comparing the derived measures to measures based on microscopic traffic simulation using the Intelligent Driver Model (IDM; Treiber, Hennecke, and Helbing 2000). The conclusion is that there is a good agreement in pattern and a rather good agreement in level between the derived measures and the simulation results for reasonable assumptions on the standard deviation of the desired speed, the segment length and the traffic flow. The derived measures should therefore not only be of theoretical interest but also be of practical use as first estimations of the performance of single lane road segments.

The verification presented in this paper considered traffic consisting of one vehicle type and the distribution of desired speeds within this type of vehicles. However, there is not only a heterogeneity between vehicles of a specific type but also between vehicle types, e.g. differences in desired speed between trucks and cars due to different speed limits and power to weight ratios. An interesting topic for future work is therefore to study the applicability of the derived measures to traffic including different types of vehicles. This can be achieved by e.g. using a multi modal density function to describe the desired speed distribution, where the modes represent different vehicle categories. In addition to the effects of the variation of desired speeds considered in this paper, effects of different mean desired speed could be studied.

The use of a well calibrated traffic simulation model (IDM; Treiber, Hennecke, and Helbing 2000; Brockfeld, Kühne, and Wagner 2005; Punzo and Simonelli 2005) for the verification allowed a systematic analysis of the derived measures. Validation with real traffic data is nevertheless a natural continuation of the research presented. Part of such a validation study should be to further investigate practical implications of the assumptions made, i.e. instantaneous speed adaptation, negligible vehicle lengths and following headways and exponentially distributed inter-arrival times to the single lane segment. For this task representative trajectory data sets are needed.

A topic for further research is also the possibilities to extend the modelling in order to limit possible practical implications of the assumptions made. One way to compensate the assumptions on zero vehicle length and following headways might be to adjust the traffic flow with a correction factor, e.g. $\hat{q}=\frac{q}{1-q t_{h w y}}$ where $t_{h w y}$ is the average steady state following time headway. This is similar as the approach of correcting traffic flows with respect to the share of heavy vehicles using personal car equivalents (pce). Test calculations shows that such a correction factor improve the agreement with the simulation results. However, it has not been investigated how theoretical appropriate such a correction factor is. Further work is needed to elaborate on any potential correction factors and their implications on the model properties and the effect on agreement with real world traffic measures. 


\section{6. *Acknowledgement}

This work received support from Swedish National Road and Transport Research Insti-

tute (VTI) and Transport research environment with novel perspectives (TRENoP). 


\section{References}

Barceló, J., ed. 2010. Fundamentals of traffic simulation. International series in operations research and management science. New York: Springer Science+Business Media, LLC.

Bergh, T., A. Carlsson, and M. Larsson. 2003. "Swedish vision zero experience." International Journal of Crashworthiness 8 (2): 159-167.

Berry, D. A., and B. W. Lindgren. 1996. Statistics: Theory and methods. Duxbury Resource Center.

Branston, D. 1979. "Method of estimating the free speed distribution for a road." Transportation Science 13 (2): 130-145.

Brewer, M.A., S.P. Venglar, and L. Ding. 2012. "Super 2 highways in Texas - operational and safety characteristics." Transportation Research Record 2301: 46-54.

Brilon, W. 1977. "Queueing model of two-lane rural traffic." Transportation Research 11 (2): 95-107.

Brockfeld, E., R.D. Kühne, and P. Wagner. 2005. "Calibration and validation of microscopic models of traffic flow." Transportation Research Record 1934: 179-187.

Carlsson, Arne, and Andreas Tapani. 2005. "Rural Highway Design Analysis through Traffic Micro-simulation." In Proceedings of the the 5th International Symposium on Highway Capacity and Quality of Service, Yokohama.

Catbagan, J.L., and H. Nakamura. 2006. "Evaluation of performance measures for two-lane expressways in Japan." Transportation Research Record 1988: 111-118.

Catbagan, J.L., and H. Nakamura. 2008. "Desired speed distributions on two-lane highways under various conditions." Transportation Research Record 2088: 218-226.

Cowan, R.J. 1971. "A road with no overtaking." Australian Journal of Statistics 13 (2): 94-116.

Cowan, R.J. 1980. "Further results on single-lane traffic flow." Journal of Applied Probability 17: 523-531.

Daganzo, C.F. 1975. "Probabilistic structure of two-lane road traffic." Transportation Research 9 (6): 339-346.

Galin (Goldfarb), D., and B. Epstein. 1974. "Speeds and delays on two-lane roads, where passing is possible at given points of the road." Transportation Research 8 (1): 29-37.

Hoogendoorn, S.P. 2005. "Unified approach to estimating free speed distributions." Transportation Research Part B: Methodological 39 (8): 709-727.

Kesting, A, M Treiber, M Schönhof, and D Helbing. 2007. "Extending adaptive cruise control to adaptive driving strategies." Transportation Research Record 2000: 16-24.

Laval, J.A. 2006. "A macroscopic theory of two-lane rural roads." Transportation Research Part B: Methodological 40 (10): 937-944.

Lighthill, M.J., and G.B. Whitham. 1955. "On kinematic waves II: A theory of traffic flow on long crowded roads." Proceedings of the Royal Society 229 (1178): 317-345.

Luttinen, Tapio. 2001. "Percent time-spent-following as performance measure for two-lane highways." Transportation Research Record 1775: 52-59.

Munehiro, K, A Takemoto, N Takahashi, M Watanabe, and Asano M. 2012. "Performance Evaluation for Rural Two-Plus-One-Lane Highway in a Cold Snowvy Region." Transportation Research Record 2272: 161-172.

Newell, G.F. 1998. "A moving bottleneck." Transportation Research Part B: Methodological 32B (8): 531-537.

Park, B-J, k Fitzpatrick, and M Brewer. 2012. "Safety effectiveness of super 2 highways in Texas." Transportation Research Record 2280: 38-50.

Punzo, V., and F. Simonelli. 2005. "Analysis and comparison of microscopic traffic flow models with real traffic microscopic data." Transportation Research Record 1934: 53-63.

Richards, P.I. 1956. "Shock waves on the highway." Operations Research 4: 42-51.

Shiomi, Y., T. Yoshii, and R. Kitamura. 2011. "Platoon-based traffic flow model for estimating breakdown probability at single-lane expressway bottlenecks." Transportation Research Part B: Methodological 45 (9): 1314-1330.

Transportation Research Board. 2010. "Chapter 5 - Quality and Level-of-Service Concepts." In HCM2010 - Highway Capacity Manual, Vol. 1: Concepts5-11. Washington D.C., USA: Trans- 

Transportmetrica A: Transport Science single lane main file
portation Research Board of the National Academies.
Treiber, M., A. Hennecke, and D. Helbing. 2000. "Congested traffic states in empirical observa-
tions and microscopic simulations." Physical Review E - Statistical Physics, Plasmas, Fluids,
and Related Interdisciplinary Topics $62(2): 1805-1824$.
Treiber, Martin, and Arne Kesting. 2013. Traffic Flow Dynamics. Springer. https:
//login.e.bibl.liu.se/login?url=http://search.ebscohost.com/login.aspx? Transportmetrica A: Transport Science single lane main file
portation Research Board of the National Academies.
Treiber, M., A. Hennecke, and D. Helbing. 2000. "Congested traffic states in empirical observa-
tions and microscopic simulations." Physical Review E - Statistical Physics, Plasmas, Fluids,
and Related Interdisciplinary Topics $62(2): 1805-1824$.
Treiber, Martin, and Arne Kesting. 2013. Traffic Flow Dynamics. Springer. https:
//login.e.bibl.liu.se/login?url=http://search.ebscohost.com/login.aspx? Transportmetrica A: Transport Science single'lane main'file
portation Research Board of the National Academies.
Treiber, M., A. Hennecke, and D. Helbing. 2000. "Congested traffic states in empirical observa-
tions and microscopic simulations." Physical Review E - Statistical Physics, Plasmas, Fluids,
and Related Interdisciplinary Topics $62(2): 1805-1824$.
Treiber, Martin, and Arne Kesting. 2013. Traffic Flow Dynamics. Springer. https:
//login.e.bibl.liu.se/login?url=http://search.ebscohost.com/login.aspx? Transportmetrica A: Transport Science single lane main file
portation Research Board of the National Academies.
Treiber, M., A. Hennecke, and D. Helbing. 2000. "Congested traffic states in empirical observa-
tions and microscopic simulations." Physical Review E - Statistical Physics, Plasmas, Fluids,
and Related Interdisciplinary Topics $62(2): 1805-1824$.
Treiber, Martin, and Arne Kesting. 2013. Traffic Flow Dynamics. Springer. https:
//login.e.bibl.liu.se/login?url=http://search.ebscohost.com/login.aspx? Transportmetrica A: Transport Science single lane main file
portation Research Board of the National Academies.
Treiber, M., A. Hennecke, and D. Helbing. 2000. "Congested traffic states in empirical observa-
tions and microscopic simulations." Physical Review E - Statistical Physics, Plasmas, Fluids,
and Related Interdisciplinary Topics $62(2): 1805-1824$.
Treiber, Martin, and Arne Kesting. 2013. Traffic Flow Dynamics. Springer. https:
//login.e.bibl.liu.se/login?url=http://search.ebscohost.com/login.aspx? Transportmetrica A: Transport Science single lane main 'file
portation Research Board of the National Academies.
Treiber, M., A. Hennecke, and D. Helbing. 2000. "Congested traffic states in empirical observa-
tions and microscopic simulations." Physical Review E - Statistical Physics, Plasmas, Fluids,
and Related Interdisciplinary Topics $62(2): 1805-1824$.
Treiber, Martin, and Arne Kesting. 2013. Traffic Flow Dynamics. Springer. https:
//login.e.bibl.liu.se/login?url=http://search.ebscohost.com/login.aspx? direct $=$ true\&db=edb\&AN $=93907720 \&$ site $=$ eds -1 ive. 


\section{Appendix A.}

With fixed $u$ and $v$, the distance to overtaking is proportional to the time between arrivals. Let $X$ be this time, with exponential distribution and parameter $\theta$. The probability density function, pdf, is $f_{X}(x)=\theta e^{-\theta x}$. Let $S=g(X)=k X$ for a constant $k>0$ be the distance to overtaking. For this transformation, $f_{Y}(y)=f_{X}\left(g^{-1}(y)\right) \frac{d g^{-1}(y)}{d y}$ (Berry and Lindgren 1996, p 177). We get $f_{Y}(y)=\theta e^{-\theta \frac{y}{k}} \cdot \frac{1}{k}=\frac{\theta}{k} e^{-\frac{\theta}{k} y}$. This is recognized as the pdf of an exponential distribution with parameter $\frac{\theta}{k}$.

Let $X$ be the distance to where a vehicle is overtaken. This is an exponentially distributed random variable with parameter $\theta$. The cumulative density function, cdf, of $X$ is $F_{X}(x)=1-e^{-\theta x}$. Let $Y$ be the distance to where another vehicle is overtaken. This is another exponentially distributed random variable, independent of $X$, with parameter $\lambda$.

The distance to the first of these two overtakings is $S=\min (X, Y)$. The cdf of $S$ is $F_{S}(s)=P(\min (X, Y) \leq s)=P((X \leq s) \cup(Y \leq s))=1-P((X>s) \cap(Y>s))=$ $1-\left(1-F_{X}(s)\right)\left(1-F_{Y}(s)\right)=1-\left(1-\left(1-e^{-\theta s}\right)\right)\left(1-\left(1-e^{-\lambda s}\right)\right)=1-e^{-\theta s} e^{-\lambda s}=1-e^{-(\theta+\lambda) s}$. That is recognized as the cdf of an exponential distribution with parameter $\theta+\lambda$. The method can be extended to any number of variables. 